\title{
Clinical Impact of Picture Archiving and Communication Systems: Evaluation of a Prototype System
}

\author{
John A. Worrell, Charles F. Federspiel, Jeffrey L. Creasy, David R. Pickens III, Kathy Higginbotham, \\ James A. Patton, and C. Leon Partain
}

\begin{abstract}
Opinion surveys were gathered before and 6 months after installation of a prototype picture archiving and communication system (PACS) (PACS/1, Siemens Medical Systems, (selin, NJ). Median turnaround times and the percent of delayed or missing reports were calculated for 1,026 baseline and 8,438 follow-up studies at 6 months. Neuroradiological (neuro) computed tomography (CT) used PACS, while neuro magnetic resonance (MR), body $C T$, and body MR served as controls. The opinion surveys showed improved service in all categories, including those not directly affected by PACS. PACS images favorably impressed $86 \%$ of respondents, but most considered the system too slow, unreliable, and the storage capacity too low. A majority of $81 \%$ recommended against purchase of PACS now. There was an overall increase in the median report turnaround time for both neuro $\mathrm{CT}$ and the controls. Neuro CT showed a $41 \%$ decrease in delayed or missing reports, but controls also showed similar decreases. The effects of this prototype PACS on turnaround time or on report delivery could not be distinguished from section-wide changes in CT and MR services. Future improvements in PACS should vigorously address increased speed, reliability, and storage capacity.
\end{abstract}

Copyright $\odot 1992$ by W.B. Saunders Company

KEY WORDS: PACS, teleradiology.

$\mathbf{I}^{\mathrm{N}}$ NTERPRETATION and archiving of radiographic images traditionally has been accomplished using the medium of photographic film. This film-based system has served radiology well as a simple and relatively inexpensive means of capturing and storing an enormous amount of diagnostic radiographic information. Any modality attempting to replace this entrenched and successful method of image interpretation and archiving will face a formidable task in proving itself the better system. It is the purpose of this

From the Departments of Radiology \& Radiological Sciences and Preventive Medicine, Vanderbilt University Medical Center, Nashville, TN.

Supported in part by a grant from Siemens Medical Systems, Iselin, $N J$.

Address reprint requests to John A. Worrell, MD, Department of Radiology and Radiological Sciences, Vanderbilt University Medical Center, Nashville, TN 37232-2675.

Copyright $\odot 1992$ by W.B. Saunders Company 0897-1889/92/0502-0006\$03.00/0 study to evaluate the clinical impact of a prototype picture archiving and communication systems (PACS) on the computed tomography (CT) and magnetic resonance (MR) services at a university hospital.

\section{MATERIALS AND METHODS}

Installation of a Siemens PACS/1 PACS unit was completed April 2, 1990. The system consists of a diagnostic reporting console (DRC/80) with eight monitors in a four-over-four arrangement. A magnetic disk provides approximately 750 Mbytes of rapidly accessible storage, while a dual-drive optical disk system is used for permanent archiving. The system is controlled by a MicroVAX II host computer and is linked by a local area network to a Siemens Magnetom MRI facility, two Siemens Somatom DRH CT units, and an additional Siemens Somatom Plus CT unit.

During the study, 1,675 MRI examinations were completed for an average of 10.9 per day. The three CT units generated 7,789 studies, an average of 50.9 per day. Neuroradiological examinations comprised $82.8 \%$ of the total MR workload and $74 \%$ of all CT studies. Because the daily workload frequently exceeded the storage capacity of the magnetic disk, neuro CT examinations were prioritized for routine PACS storage.

Baseline opinion surveys of radiologists and their clinical colleagues most likely to be affected by the PACS (neurosurgery, neurology, emergency medicine, and otolaryngology) regarding the functions of the CT and MR services as a whole were carried out in the month prior to installation. Data on report turnaround times and the percentage of missing or delayed reports for this same baseline period were gathered through the radiology information system (DECrad, Digital Equipment Corporation, Maynard, MA). Missing or delayed reports were defined as those not accessed in the DECrad system at the time of data collection, 30 days after the last examination was performed. The surveys and data collection were repeated 6 months later after a period of familiarization with the PACS. The follow-up study was conducted during the months of August through November, 1990. An additional opinion survey of the impact of PACS on the radiologists and technologists actually using the PACS also was carried out after this 6-month time period. Turnaround times were measured in minutes from the completion of the examination until transcription of the radiological interpretation. The opinion surveys required graded responses scaled from 1 to 10 , with 10 representing the most favorable response.

The DRC/80 was physically located in the neuroradiology CT reading area. The radiologist could choose to interpret examinations on either (or both) the PACS $\mathrm{DRC} / 80$ or the standard film alternator display. Although network links with body $\mathrm{CT}$, body MR, and neuro MR 
facilities were present, only the neuroradiologists made use of the PACS facility for diagnostic interpretation because of the physical location of the DRC/ 80 interpretive console, as well as the selective priority given to neuro CT studies.

Data were analyzed using a commercially available software package (SAS Institute, Carey, NC). Arithmetic means were calculated for the opinion parameters. The missing or delayed reports were calculated as the percent of the total reports for each of the categories of examination. The turnaround times were analyzed using the median times in addition to the arithmetic and geometric means. Because of the skewed nature of the arithmetic means, analysis of variance (ANOVA) of the normally distributed logarithms of the turnaround times was used to test significance of any differences among their geometric means. Differences were considered significant at the $P<0.01$ level.

\section{RESULTS}

The opinion surveys included 25 respondents, 8 of whom answered both the initial and the follow-up questionnaires (Tables 1 and 2). The results are depicted in graphic form (Figs 1 and 2) and are summarized in Fig 3 and Table 3.

Three parameters of the CT and MR service as a whole that showed little change were the prompt scheduling of routine cases, the average in-room scan time of emergency cases, and the overall system reliability. The overall system reliability had the lowest average score of any of these parameters, averaging 3.73 (out of 10) before and 4.06 after PACS. The in-room scan

Table 1. Opinion Questionnaire of CT and MR Services as a Whole

\begin{tabular}{|c|c|c|c|c|c|c|c|c|c|c|}
\hline \multicolumn{11}{|c|}{$\begin{array}{l}\text { Please circle your perception of the } C T \text { and } M R \text { services at } \\
\text { Vanderbilt, } 1=\text { worst negative, } 5=\text { neutral, } 10=\text { best posi- } \\
\text { tive }\end{array}$} \\
\hline \multicolumn{11}{|l|}{ Prompt routine scheduling } \\
\hline of cas & 1 & 2 & 3 & 4 & 5 & 6 & 7 & 8 & 9 & 10 \\
\hline \multicolumn{11}{|l|}{ Prompt initiation of ER } \\
\hline $\operatorname{cas}$ & 1 & 2 & 3 & 4 & 5 & 6 & 7 & 8 & 9 & 10 \\
\hline \multicolumn{11}{|l|}{ Emergency in-room scan } \\
\hline time & 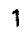 & 2 & 3 & 4 & 5 & 6 & 7 & 8 & 9 & 10 \\
\hline \multicolumn{11}{|l|}{ Prompt access to current } \\
\hline images & 1 & 2 & 3 & 4 & 5 & 6 & 7 & 8 & 9 & 10 \\
\hline \multicolumn{11}{|l|}{ Prompt access to other } \\
\hline modality images & 1 & 2 & 3 & 4 & 5 & 6 & 7 & 8 & 9 & 10 \\
\hline \multicolumn{11}{|l|}{ Availability of any consult- } \\
\hline ing radiologist & 1 & 2 & 3 & 4 & 5 & 6 & 7 & 8 & 9 & 10 \\
\hline \multicolumn{11}{|l|}{ Availability of a neuroradi- } \\
\hline ologist consultation & 1 & 2 & 3 & 4 & 5 & 6 & 7 & 8 & 9 & 10 \\
\hline \multicolumn{11}{|l|}{ Prompt report turnaround } \\
\hline time & 1 & 2 & 3 & 4 & 5 & 6 & 7 & 8 & 9 & 10 \\
\hline Overall system reliability & 1 & 2 & 3 & 4 & 5 & 6 & 7 & 8 & 9 & 10 \\
\hline \multicolumn{11}{|l|}{ Overall satisfaction with } \\
\hline CT and MR services & 1 & 2 & 3 & 4 & 5 & 6 & 7 & 8 & 9 & 10 \\
\hline
\end{tabular}

Table 2. Opinion Questionnaire of the PACS Itself

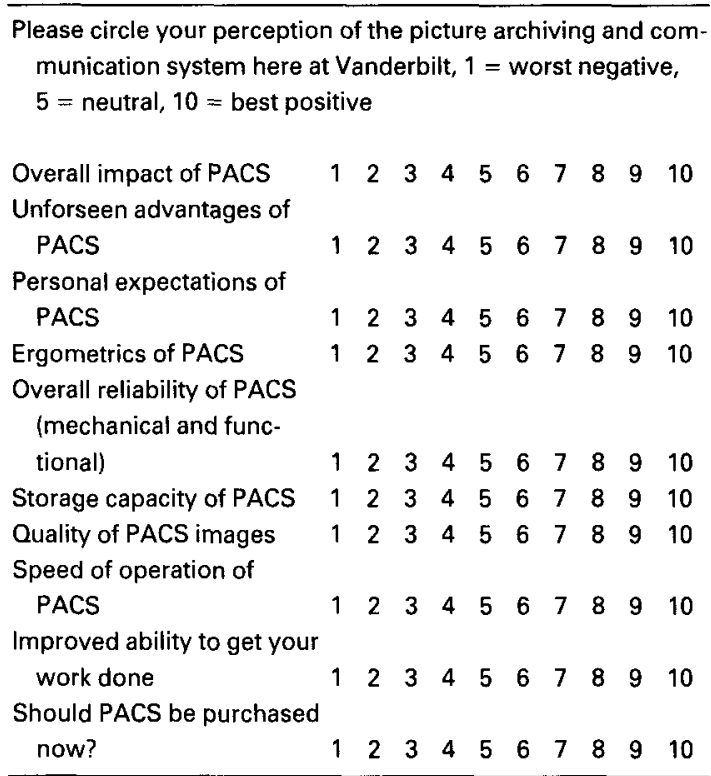

times for emergencies, on the other hand, had the highest scores, with an average of 7.59 before and 8.14 after PACS.

All of the other parameters of the CT and MR service as a whole showed an average score improvement of at least 0.9 . Access to the diagnostic images improved from 4.48 to 5.59 . Availability of other modality images improved even more (from 2.68 to 4.29 ), but these images were not stored on PACS. An improvement in the availability of any radiologic consultation improved from 3.46 to 4.43 , with the improvement in the availability of subspecialty neuroradiology consultation improving even more (from 4.86 to 6.88 ). A summary statistic, overall satisfaction, improved from 3.92 to 5.71 .

In summary, every parameter of the CT and MR service as a whole improved after the installation of PACS, with some of these improvements clearly not related to PACS. However, there were important trends toward better scores, which were also were noted in the PACS-related parameters.

The data regarding the opinion surveys of the PACS itself are presented in graphic form (Figs 4, 5, and 6). Several trends are seen here. First, the most positive opinion was noted in the quality of the images on the PACS, which was reported above expectation by 12 of $14(86 \%)$ of the respondents, with 6 of $14(43 \%)$ scoring the images as a 9 or 10 . 
Turnaround Time

(Before)

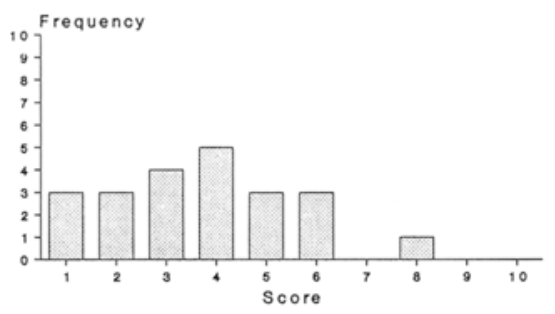

Turnaround Time

(After)

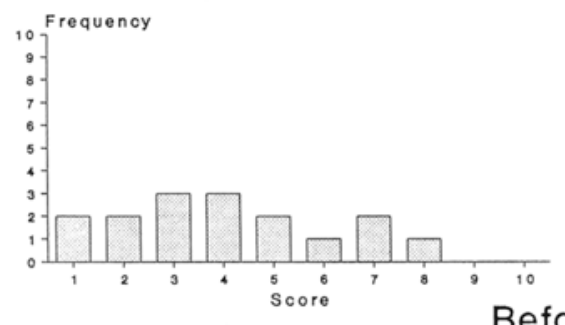

Overall Satisfaction

(Before)

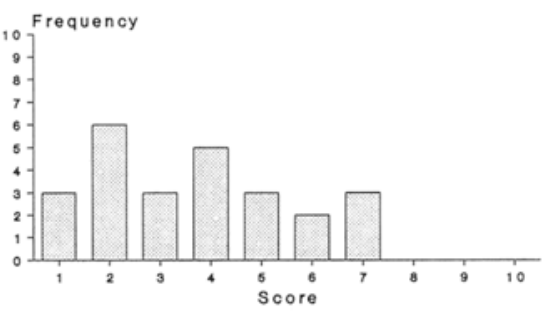

Overall Satisfaction

(After)

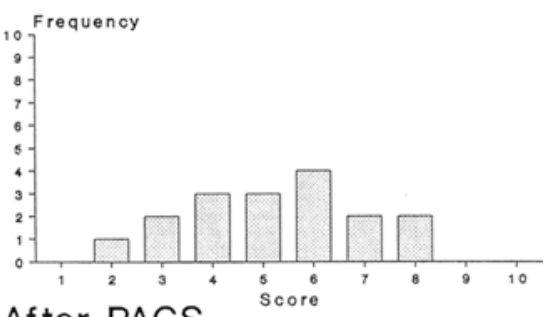

Fig 1. Opinion survey results of radiology system as a whole.

Several of the other areas, though, revealed more negative opinions. Respondents considered the system reliability low (13 of 19$)$, the storage capacity inadequate (13 of 17), and the speed of operation too slow (13 of 16). They did not report an increase in productivity (13 of 16), and the overall impact of PACS was rated as below expectations by 10 of 14 respondents. Thirteen of 16 respondents did not recommend purchase of PACS at present, with 7 of those ranking this option as the worst negative case (Fig 6).

A total of 1,026 CT and MR examination reports was collected for the baseline month,
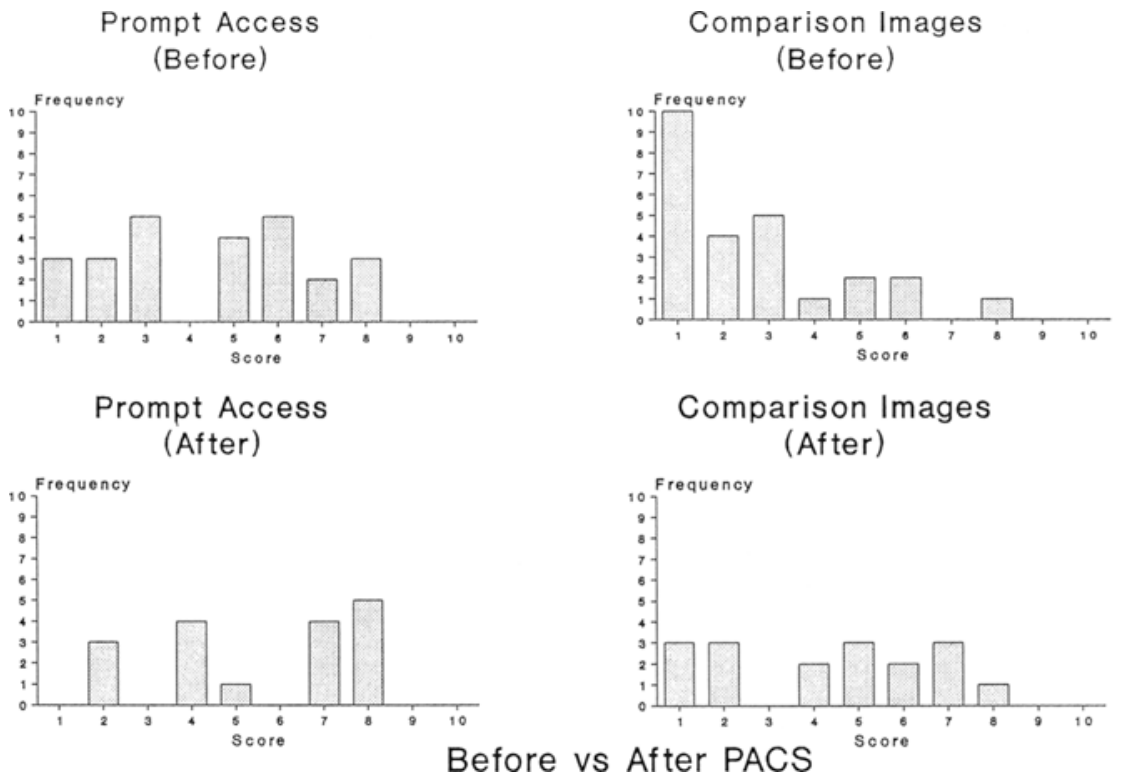

Fig 2. Opinion survey results of radiology system as a whole. 


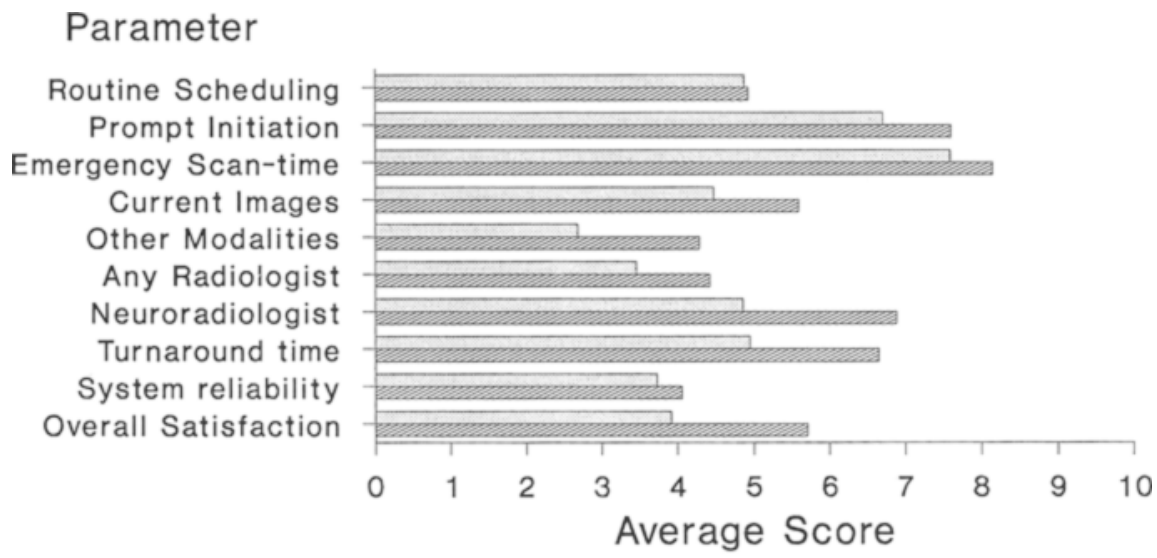

Fig 3. Average opinion survey scores of the $C T$ and MR services. $\square$, before PACS, $\square$, after PACS.

with an additional 8,438 reports for the 3 months of follow-up, which began 6 months after installation. Neuro CT comprised $49 \%$ of all baseline and $62 \%$ of all follow-up studies. By contrast, body MR had the smallest contribution, with only $5 \%$ of baseline and $3 \%$ of all follow-up studies.

Results of report turnaround times (in minutes) and for examinations completed but with delayed or missing reports are summarized in Table 4 and Fig 7. Median as well as arithmetic and geometric mean times are included because of the skewed nature of the frequency distributions.

These data show an increase in the median turnaround time for all categories of reports (both PACS as well as non-PACS-related) from the April to the August through November

Table 3. Average Scores from Opinion Surveys Evaluating CT and MR Services Taken Before and 6 Months After PACS Installation

\begin{tabular}{lcc}
\hline \multicolumn{1}{c}{ Opinion Parameter } & $\begin{array}{c}\text { Before } \\
\text { (Standard } \\
\text { Deviation) }\end{array}$ & $\begin{array}{c}\text { After } \\
\text { (Standard } \\
\text { Deviation) }\end{array}$ \\
\hline Prompt routine scheduling & $4.88(2.38)$ & $4.93(2.79)$ \\
Prompt initiation of ER cases & $6.70(2.44)$ & $7.60(2.06)$ \\
Emergency scan time & $7.59(1.56)$ & $8.14(1.46)$ \\
Access to current images & $4.48(2.31)$ & $5.59(2.32)$ \\
Access to other modality images & $2.68(1.97)$ & $4.29(2.39)$ \\
Availability of any radiologist & $3.46(2.06)$ & $4.43(2.41)$ \\
Availability of neuroradiologist & $4.86(2.48)$ & $6.88(2.32)$ \\
Report turnaround time & $4.95(2.46)$ & $6.65(2.12)$ \\
Overall system reliability & $3.73(1.86)$ & $4.06(2.14)$ \\
Overall satisfaction & $3.92(2.21)$ & $5.71(1.76)$ \\
\hline
\end{tabular}

NOTE. Twenty-five respondents in baseline survey, 17 in follow-up. Responses rated on a scale of 1-10 with 10 the best possible circumstance. period (Table 4, Fig 7). Analysis of variance of the logarithims of the turnaround times showed that the increases in the geometric means from the baseline to the follow-up study were statistically significant at the $P<0.01$ level for all comparisons except for body MR $(P=0.15)$. No objective improvement in turnaround time was documented in any category after PACS was installed.

The number of examinations with delayed or missing reports also was studied (Table 5, Fig 8 ). These data document a slight increase in the percentage of examinations with a delayed or missing report in both neuro MR and in body CT. There was a decrease in the number of delayed or missing reports seen in neuro CT (the most intensive use of PACS), but even more dramatic effects were seen in other areas where the percentage of delayed or missing reports dropped from $23 \%$ to $8.3 \%$ for body MR and from $30 \%$ to $20.7 \%$ for body CT.

\section{DISCUSSION}

As successful as the film-based interpretation and archiving system has been for radiology and medical imaging, it also has inherent disadvantages. ${ }^{1}$ The plain film requires a queue when more than one party is interested in viewing the image, and conflicts may result when prioritization is necessary. Photographic film can be lost or misplaced and must be physically transported for viewing at remote sites. Although relatively inexpensive on a per-copy basis, costs are significant in a large volume operation, especially when the costs of photographic copy film and 
Impact of PACS

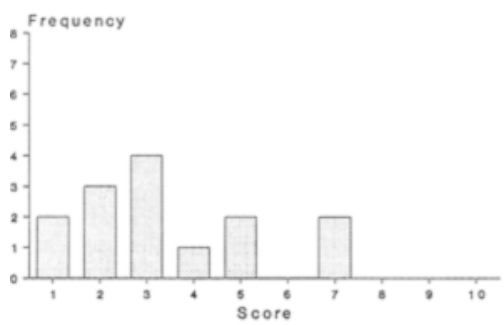

Expectations of PACS

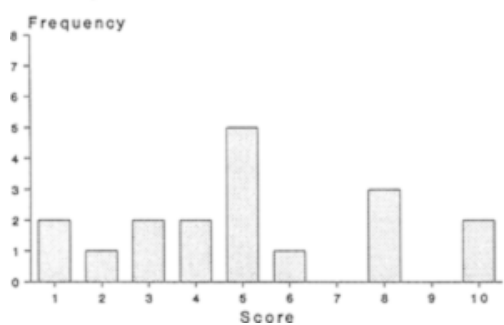

Unforseen Advantages

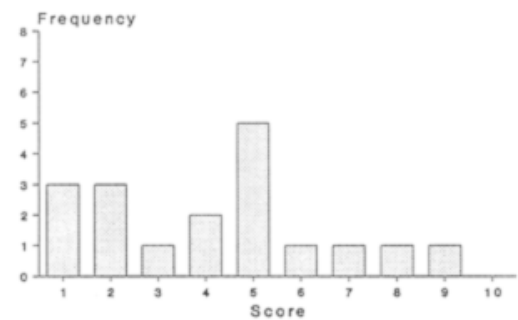

Ergometrics of PACS

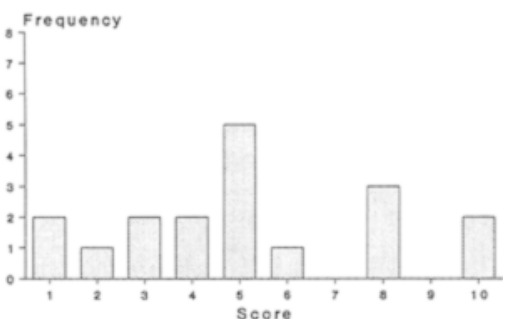

Fig 4. PACS opinion survey results.

storage are accounted. The trend toward decentralization of radiology departments away from main hospitals, together with the rise in outpatient imaging and treatment facilities require efficient, rapid transfer of medical images. It is for these reasons that the PACS alternative must be seriously evaluated.

This evaluation began with opinion surveys of the performance of the CT and MR sections of the radiology department before and 6 months

Reliability of PACS

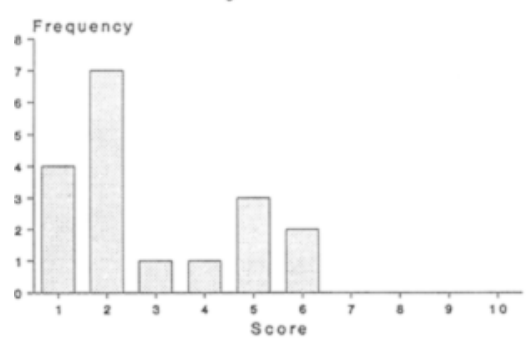

Quality of PACS Images

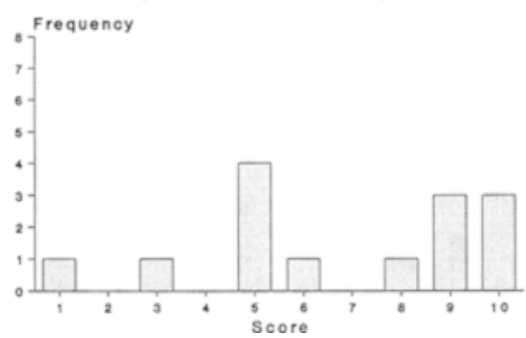

after PACS had been installed. These data show an overall improvement in the perception of service during this period, but some of the improvements are in areas clearly not affected by PACS and probably reflect departmentalwide effects of an intensive effort to improve service. Although trends toward better scores were noted in parameters of departmental service that PACS could be expected to impact, no obvious effect from the PACS was noted.

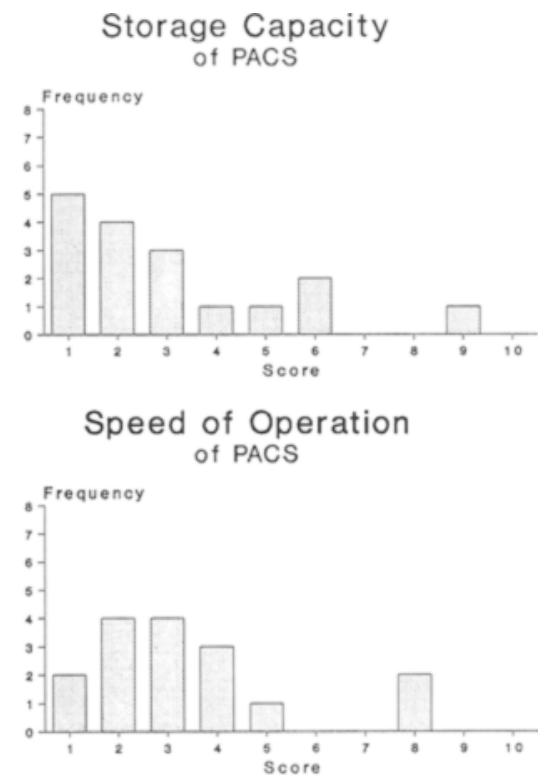

Fig 5. PACS opinion survey results. 
Improved Ability

to Get Work Done

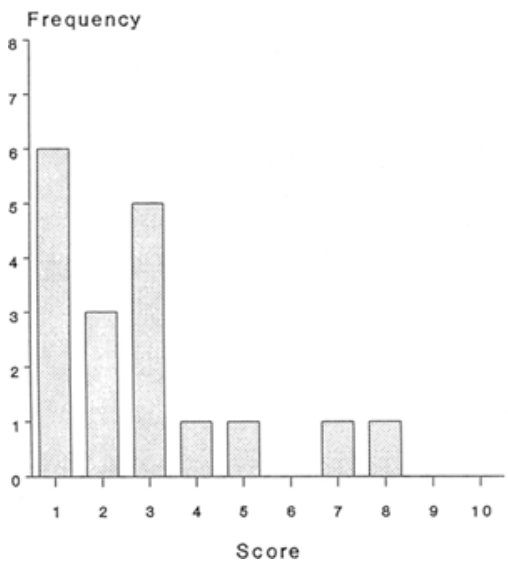

Should PACS

Be Purchased?

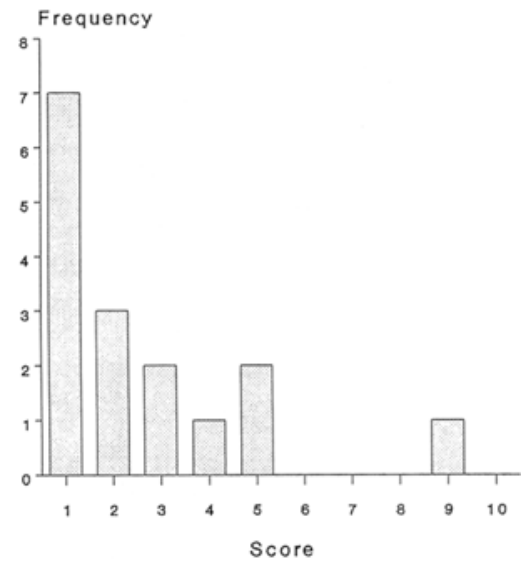

Fig 6. PACS opinion survey results.

The opinion surveys among the users of the PACS regarding the PACS system itself revealed several important trends. The positive opinions regarding the PACS image quality corroborate the work of Berbaum et $\mathrm{al}^{2}$ who compared the interpretation of body CT studies on a PACS system against standard hardcopy images and found the cases depicted equally well in $75 \%$ of cases. ${ }^{2}$ They judged PACS imaging better in the remaining $25 \%$. The most negative opinions, on the other hand, were related to system reliability, storage capacity, speed of operation, and increased productivity.

There were relatively few service interruptions due to the PACS itself. Underlying these negative opinions regarding the ability of the PACS to function quickly and efficiently was the storage capacity of the magnetic disk. Magnetic resonance imaging examinations average 50 digital images per study. ${ }^{3}$ With a $256 \times 256 \times$

Table 4. Turnaround Times for Reports Before and After PACS Installantion

\begin{tabular}{|c|c|c|c|c|c|c|c|c|}
\hline & \multicolumn{2}{|c|}{ Body MR } & \multicolumn{2}{|c|}{ Neuro MR } & \multicolumn{2}{|c|}{ Body CT } & \multicolumn{2}{|c|}{ Neuro CT } \\
\hline & Before & After & Before & After & Before & After & Before & After \\
\hline$N$ & 56 & 232 & 223 & 1,164 & 249 & 1,774 & 498 & 2,098 \\
\hline$\%$ & 5 & 3 & 21 & 14 & 24 & 21 & 49 & 62 \\
\hline Median & 1,211 & 1,441 & 1,204 & 1,548 & 1,084 & 1,292 & 1,274 & 1,576 \\
\hline \multicolumn{9}{|c|}{ Arithmetic } \\
\hline mean & 1,519 & 1,964 & 1,469 & 2,039 & 2,247 & 1,904 & 4,494 & 2,862 \\
\hline \multicolumn{9}{|c|}{ Geometric } \\
\hline mean & 1,149 & 1,391 & 1,127 & 1,597 & 955 & 1,176 & 1,240 & 1,729 \\
\hline
\end{tabular}

Abbreviation: $\mathbf{N}$, number of examinations. 12-bit matrix, 10.9 studies per day create an average of 71.4 Mbytes of data. Computed tomography averages 30 digital images per examination. ${ }^{3}$ The $512 \times 512 \times 12$-bit image and average of 50.9 daily studies add another 801 Mbytes. Together, CT and MRI averaged over 870 Mbytes of information daily. With peak loads and the requirement for holding studies several days for review by clinical colleagues, the 750 Mbyte capacity of the magnetic disk was overwhelmed. This problem was complicated by a lack of automatic long-term archiving to optical disk of studies no longer of current interest. This problem was anticipated, but selective priority use of the neuro CT cases for PACS provided only a partial solution. This reduced the average daily volume of data to 593 Mbytes but introduced the additional problem, particularly for the attending clinicians, of deciding which cases might actually be in the PACS.

Perhaps the most revealing question regarded the recommendation to purchase PACS now. The majority of respondents registered a negative opinion, with 7 of 13 expressing this negative recommendation in the strongest possible category.

The objective measurement of the impact of PACS began by comparing baseline studies of the report turnaround times both before as well as 6 months after PACS was installed. The PACS DRC/80 console was physically located in the neuroradiology section, and selective 


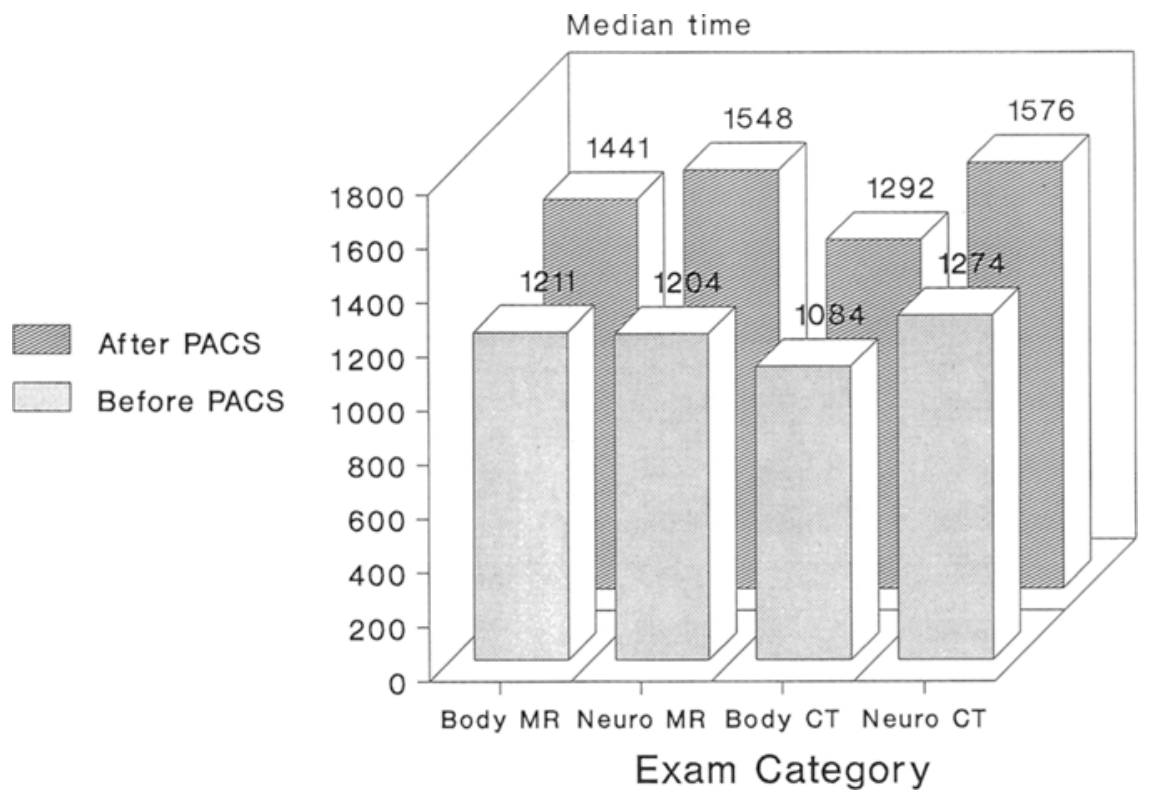

Time in minutes

Fig 7. Median turnaround times for reports.

priority use was assigned to neuro CT. PACS was effectively not used for diagnostic interpretation by the body CT and MR sections, allowing these sections to be used as controls. An increase in median report turnaround times was noted in all four of the surveyed areas, reflecting CT and MR section-wide effects rather than a PACS effect.

The next objective measurement was in the important area of examinations with delayed or missing reports. Data collection was accomplished 30 days after the last examination had been performed, and reports not accessible on DECrad were considered delayed or missing at this point. The heaviest user of PACS, neuro CT, did show a dramatic decrease from $20.7 \%$ to $12.3 \%$ missing or delayed reports after the installation of PACS, but this effect also was

Table 5. Percent Delayed or Missing Reports Before and After PACS Installation

\begin{tabular}{|c|c|c|c|c|c|c|c|c|}
\hline & \multicolumn{2}{|c|}{ Body MR } & \multicolumn{2}{|c|}{ Neuro MR } & \multicolumn{2}{|c|}{ Body CT } & \multicolumn{2}{|c|}{ Neuro CT } \\
\hline & Before & After & Before & After & Before & After & Before & After \\
\hline $\mathbf{N}$ & 73 & 253 & 251 & 1,326 & 293 & 2,221 & 628 & 2,391 \\
\hline NDel & 17 & 21 & 28 & 162 & 88 & 447 & 130 & 293 \\
\hline$\%$ Del & 23 & 8.3 & 11.1 & 12.2 & 30 & 20.1 & 20.7 & 12.3 \\
\hline
\end{tabular}

Abbreviations: $N$, number of examinations in this time period; NDel, Number with delayed or missing reports; \% NDel, percent of examinations with delayed or missing reports. seen in another high volume user, body CT. Although body CT did not use PACS, the missing or delayed reports from that section dropped from $30 \%$ to $20.1 \%$ (Fig 8). Again, the improvements here are thought due to $\mathrm{CT}$ and MR section-wide effects rather than any effect of PACS. During this time period, an intensive effort was under way in all sections of the radiology department to find and report delayed films. This search offers the best explanation for the increase in median report times together with the decrease in missing or delayed reports. Films that were previously missing or not reported were now being found and reported later, but still within the 30-day delay before data collection.

In conclusion, the prototype PACS evaluated at Vanderbilt University offers superb diagnostic images, but neither subjective nor objective data support a positive impact on our department. The major shortcomings were in the system reliability and storage capacity. Whereas there were relatively few service interruptions due to the PACS itself, the flow of information was choked at the magnetic disk. Future design must vigorously address the enormous volume of data that must be accommodated rapidly and reliably. The challenges outlined by Templeton 


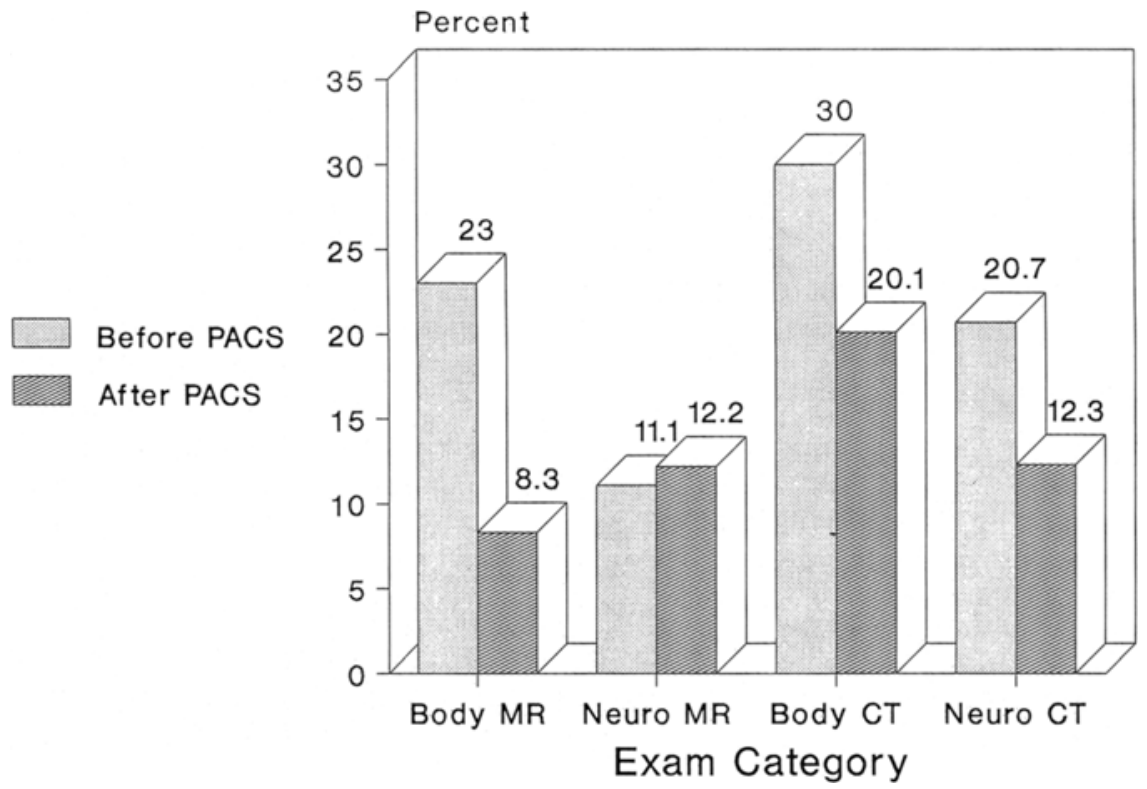

\section{Before vs After PACS}

Fig 8. Percent delayed or missing reports.

et al in 1988 remain. ${ }^{4}$ These include the need for fast, inexpensive, large buffer storage; adequate software for operating and managing the system; and an educational program for training the medical staff in the use of these devices. As these obstacles are inevitably surmounted, PACS shall effectively challenge the film-based interpretation and archival system currently in use.

\section{REFERENCES}

1. Cox GG, Templeton AW, Dwyer SJ: Digital image management: Networking, display, and archiving. Radiol Clin North Am 24:37-54, 1986

2. Berbaum KS, Franken EA, Honda H, et al: Evaluation of a PACS workstation for assessment of body CT studies. I Comput Assist Tomogr 14:853-858, 1990
3. Dwyer SJ, Templeton AW, Batnitzky S: Teleradiology: Costs of hardware and communications. AJR 156:12791282,1991

4. Templeton AW, Cox GG, Dwyer JD III: Digital image management networks: Current status. Radiology 169:193199,1988 